\title{
Idiopathic Normal Pressure Hydrocephalus has a Different Cerebrospinal Fluid Biomarker Profile from Alzheimer's Disease
}

\author{
Naoto Jingami ${ }^{\mathrm{a}, \mathrm{b}}$, Megumi Asada-Utsugi ${ }^{\mathrm{a}, \mathrm{b}}$, Kengo Uemura ${ }^{\mathrm{a}, \mathrm{b}, \mathrm{c}}$, Rio Noto ${ }^{\mathrm{a}}$, Makio Takahashi ${ }^{\mathrm{d}, \mathrm{e}}$, \\ Akihiko Ozaki $^{\text {,ff }}$, Takeshi Kihara ${ }^{\mathrm{g}}$, Takashi Kageyama ${ }^{\mathrm{h}}$, Ryosuke Takahashi ${ }^{\mathrm{a}}$, Shun Shimohama ${ }^{\mathrm{i}, *}$ \\ and Ayae Kinoshita ${ }^{b}$ \\ ${ }^{a}$ Department of Neurology, Kyoto University Graduate School of Medicine, Kyoto, Japan \\ ${ }^{\mathrm{b}}$ Department of Human Health Sciences, Kyoto University Graduate School of Medicine, Kyoto, Japan \\ ${ }^{\mathrm{c}}$ Department of Neurology, Ishiki Hospital, Kagoshima, Japan \\ ${ }^{\mathrm{d}}$ Department of Neurology, Osaka Saiseikai Nakatsu Hospital, Osaka, Japan \\ ${ }^{\mathrm{e}}$ Department of Neurology, Osaka Redcross Hospital, Osaka, Japan \\ ${ }^{\mathrm{f}}$ Department of Neurology, Kitano Hospital, Osaka, Japan \\ ${ }^{\mathrm{g}}$ Department of Neurology, Misasagi Hospital, Kyoto, Japan \\ ${ }^{\mathrm{h}}$ Department of Neurology, Tenri Yorozu Hospital, Nara, Japan \\ ${ }^{\mathrm{i}}$ Department of Neurology, Sapporo Medical University, Sapporo, Japan
}

Accepted 12 November 2014

\begin{abstract}
The diagnosis of idiopathic normal pressure hydrocephalus (iNPH) is sometimes complicated by concomitant Alzheimer's disease (AD) pathology. The purpose of the present study is to identify an iNPH-specific cerebrospinal fluid (CSF) biomarker dynamics and to assess its ability to differentiate iNPH from AD. Total tau (t-tau), tau phosphorylated at threonine 181 (p-tau), amyloid- $\beta$ (A $\beta$ ) 42 and 40, and leucine-rich $\alpha$-2-glycoprotein (LRG) were measured in 93 consecutive CSF samples consisting of $55 \mathrm{iNPH}$ (46 tap test responders), $20 \mathrm{AD}, 11$ corticobasal syndrome, and 7 spinocerebeller disease. Levels of t-tau and p-tau were significantly decreased in iNPH patients especially in tap test responders compared to AD. Correlation was observed between Mini-Mental State Examination scores and $\mathrm{A} \beta_{42}$ in $\mathrm{AD}(\mathrm{R}=0.44)$ and mildly in iNPH $(\mathrm{R}=0.28)$. Although $\mathrm{A} \beta_{42 / 40}$ ratio showed no significant difference between iNPH and $\mathrm{AD}(p=0.08)$, the levels of $\mathrm{A} \beta_{40}$ and $\mathrm{A} \beta_{42}$ correlated positively with each other in iNPH $(R=0.73)$ but much less in $A D(R=0.26)$, suggesting that they have discrete amyloid clearance and pathology. LRG levels did not differ between the two. Thus, our study shows that although CSF biomarkers of iNPH patients can be affected by concomitant tau and/or amyloid pathology, CSF t-tau and p-tau are highly useful for differentiation of iNPH and $\mathrm{AD}$.
\end{abstract}

Keywords: Alzheimer's disease, amyloid- $\beta$, analysis of covariate, idiopathic normal pressure hydrocephalus, leucine-rich $\alpha-2-$ glycoprotein, Mini-Mental State Examination, tap test, tau phosphorylated at threonine 181, total tau

\footnotetext{
*Correspondence to: Shun Shimohama, Department of Neurology, School of Medicine, Sapporo Medical University, Address: South 1, West 16, Chuo-ku, Sapporo 060-8543, Japan. Tel.: +81 11 611 2111; Fax: +81 11622 7668; E-mail: shimoha@ sapmed.ac.jp.
}

\section{INTRODUCTION}

Idiopathic normal pressure hydrocephalus (iNPH) is a neurological disease that usually develops in the elderly with a typical triad (i.e., gait instability, urinary incontinence, and cognitive dysfunction [1]). Neuroradiologically, iNPH is characterized by enlarged 
ventricles, widening of cortical sulci, and tightening of parasagittal upper convexity. Impairment of cerebrospinal fluid (CSF) clearance has been thought to be the main cause of the disease, and the symptoms can be alleviated by appropriate shunt surgery. Therefore, precise diagnosis of iNPH is of importance.

In practice, the diagnostic differentiation of iNPH from other neurodegenerative disorders can be difficult. For example, differentiating iNPH from Alzheimer's disease (AD) is difficult when the cognitive function of the patient is highly impaired. Cognitive impairment due to iNPH initially presents as frontal lobe dysfunctions with deficits in attention, execution, and thinking. In an advanced stage of iNPH, short-term memory is impaired like that in AD. In other instances, differentiating iNPH from neurobehavioral disorders, such as Parkinson's disease and corticobasal syndrome (CBS), can be challenging. The gait of iNPH patients is characterized by small-steps and magnet-like features, resembling the parkinsonian gait. Evaluating the efficacy of anti-parkinsonian drugs might be helpful for the diagnosis, but it is not always available. Some iNPH patients are reported to have coexisting AD pathology and sometimes CBS $[2,3]$. In these cases, the efficacy of shunt surgery is limited and temporary, and surgical treatment cannot prevent the progressive clinical course of the comorbid diseases.

Tap test (TT) is useful for the diagnosis of iNPH, but the diagnosis is difficult in TT non-responders (NRs). Moreover, certain TT responders (Rs) undergone shunt surgery may still go through a progressive clinical course, despite the shunt surgery. The combination of neurological examination, neuroimaging study, and TT is sometimes not sufficient for a clear diagnosis. Additionally, quantitative estimates of overlapping $\mathrm{AD}$ pathology are almost impossible to assess with these clinical examinations. Thus, a biomarker is required in order to improve diagnostic accuracy and evaluation of iNPH.

Levels of total tau (t-tau), tau phosphorylated at threonine 181 (p-tau), and amyloid- $\beta$ (A $\beta$ ) are so far established as CSF biomarkers for AD. In previous reports, the levels of t-tau and p-tau are elevated and the $A \beta_{42 / 40}$ ratio is decreased in CSF from AD patients [4-7]. On the other hand, an agreement on changes in CSF tau has not been established in iNPH patients. Previous studies show various results of CSF tau levels in iNPH in comparison to normal subjects: increased [8], decreased [9], and not changing [2]. However, CSF tau in iNPH patients is at least lower than that in $\mathrm{AD}$. Another study showed that $\mathrm{CSF} A \beta_{42}$ is also decreased in iNPH as in AD [8]. Further, leucine-rich $\alpha$-2-glycoprotein (LRG) is reported to be highly elevated in iNPH and is believed to be an iNPH-specific candidate biomarker in CSF $[10,11]$.

Based on this evidence, we measured these CSF biomarkers and tried to find correlations between each biomarker level and the clinical symptoms in iNPH.

\section{MATERIALS AND METHODS}

\section{Subjects}

The study was undertaken in patients with iNPH, $\mathrm{AD}$, and other neurodegenerative diseases. Participants were enrolled from 2009 to 2012 from four hospitals in Japan: Kyoto University Hospital (Hp.), Saiseikai Nakatsu Hp., Kitano Hp., and Tenri Yorozu Hp. A total of 93 consecutive CSF samples were collected in this study (55 iNPH, $20 \mathrm{AD}, 11 \mathrm{CBS}$, and 7 spinocerebellar degeneration (SCD)). The demographics and clinical characteristics of subjects in each group are shown in Table 1.

Written informed consent for this study was obtained from each patient. Each patient underwent a standardized neurological evaluation. iNPH patients who fulfilled the diagnostic criteria of probable iNPH by the guidelines were selected including ventriculomegaly with Evans index $(\mathrm{EI})>0.3$ on a computed tomography imaging study [12]. All of the iNPH patients underwent TT, and TT Rs was defined as one whose walking speed increased more than $10 \%$. Those who did not respond were defined as NRs. Among the 55 iNPH patients, $46(83.6 \%)$ were Rs (Table 1). An $\mathrm{AD}$ diagnosis was based on current international clinical criteria [13]. Each iNPH and AD patient was scored with the Mini-Mental State Examination (MMSE).

\section{Biomarker levels in CSF samples (t-tau, p-tau, $A \beta_{42 / 40}$ ratio, $L R G$ )}

Levels of t-tau, p-tau, $A \beta_{42 / 40}$ ratio, and LRG in the CSF were measured with an enzyme-linked immunosorbent assay according to the manufacturer's protocol; Tau (Total) Human ELISA Kit - Novex, Phospho-Tau (181P) - Innotest, Human Amyloid $\beta$ (142) Assay Kit - IBL, Human Amyloid $\beta$ (1-40) Assay Kit - IBL, and Human LRG Assay Kit - IBL.

\section{Statistical analyses}

Comparisons among groups were performed using Chi-square test. Analysis of covariance (ANCOVA) was additionally used to compare the levels of each 


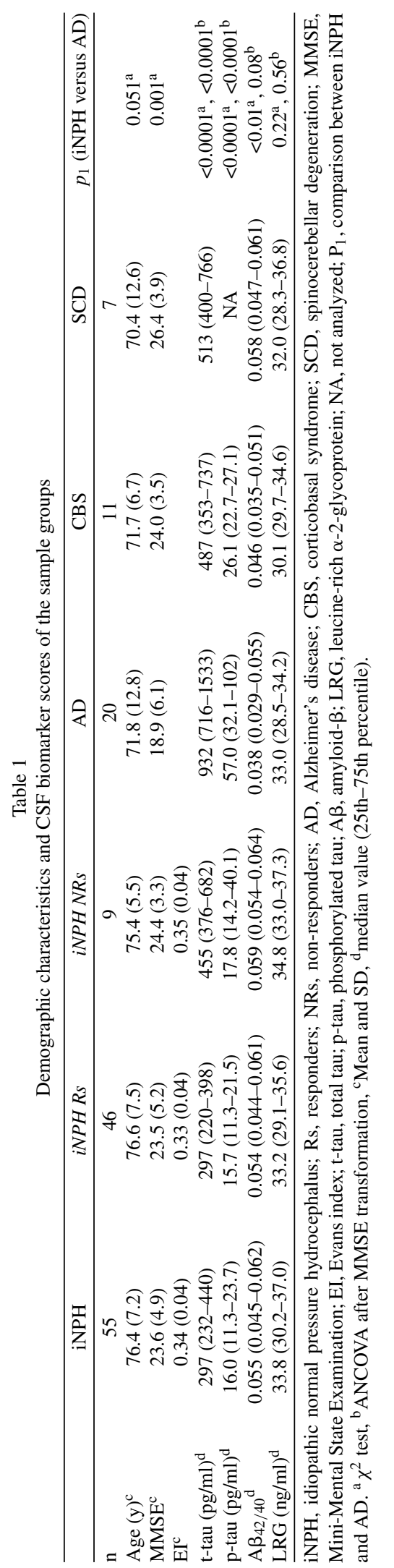


biomarker between iNPH and AD on assessments after adjustment for each individual MMSE score. Optimal sensitivity and specificity of each biomarker were determined by the receiver operating characteristic (ROC) curve analysis. Results are indicated as median and 25 th-75th percentile. Statistical significance was defined at $p<0.05$.

\section{RESULTS}

The CSF biomarker scores of each group are shown in Fig. 1 and Table 1. ROC analysis results of each biomarker for differentiating iNPH from AD are shown in Fig. 2 and Table 2.
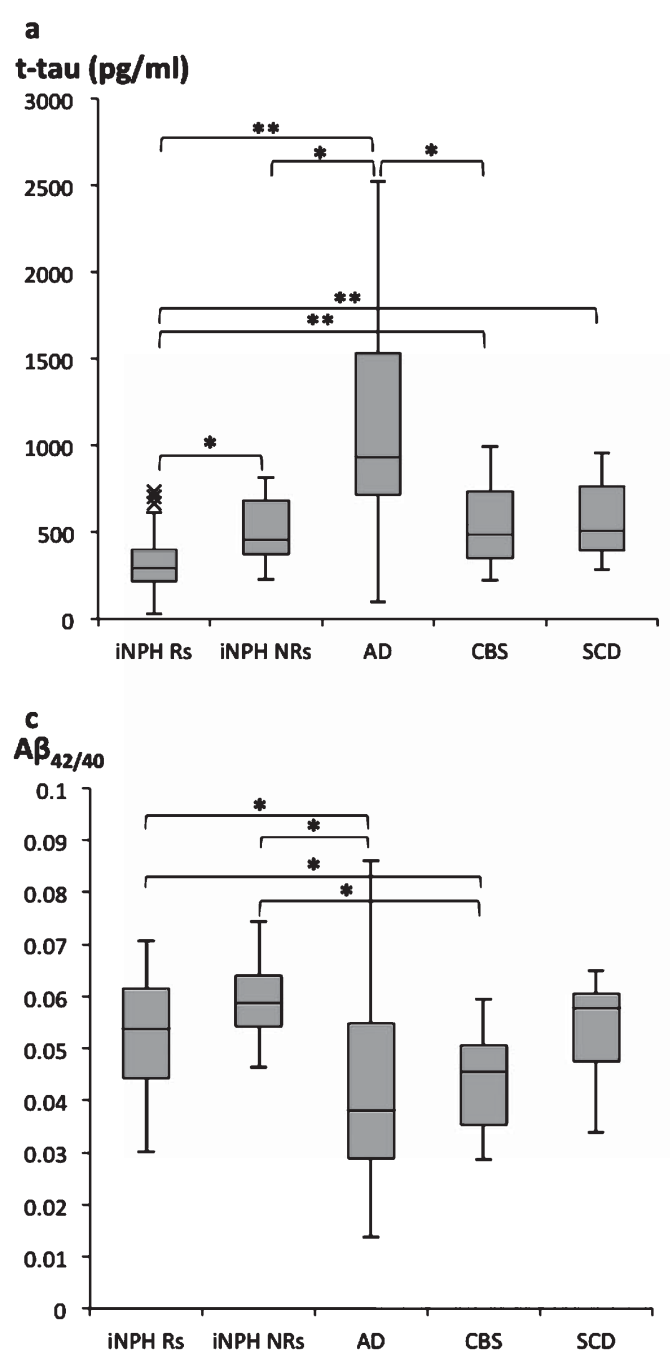

Levels of t-tau and p-tau (pg/ml) were significantly lower in iNPH patients than those in AD patients ( $p<0.0001, p<0.0001$, respectively) (Fig. 1a, b). These differences had great deals of diagnostic accuracies, with the area under the curves (AUC) 0.90 and 0.91 , with optimal threshold values of 766 (sensitivity $75 \%$, specificity 98\%) and 24.4 (sensitivity 95\%, specificity 74\%), respectively (Fig. 2). In the iNPH patients, levels of t-tau and p-tau were lower in the Rs subgroup than in the NRs subgroup $(p<0.05, p=0.06$, respectively). Levels of t-tau in Rs were lowest with significance compared with rest of the groups (Fig. 1a, b). The levels of t-tau and p-tau strongly correlated with each other in $\mathrm{AD}(\mathrm{R}=0.90)$ and iNPH NRs $(\mathrm{R}=0.91)$

\section{b}
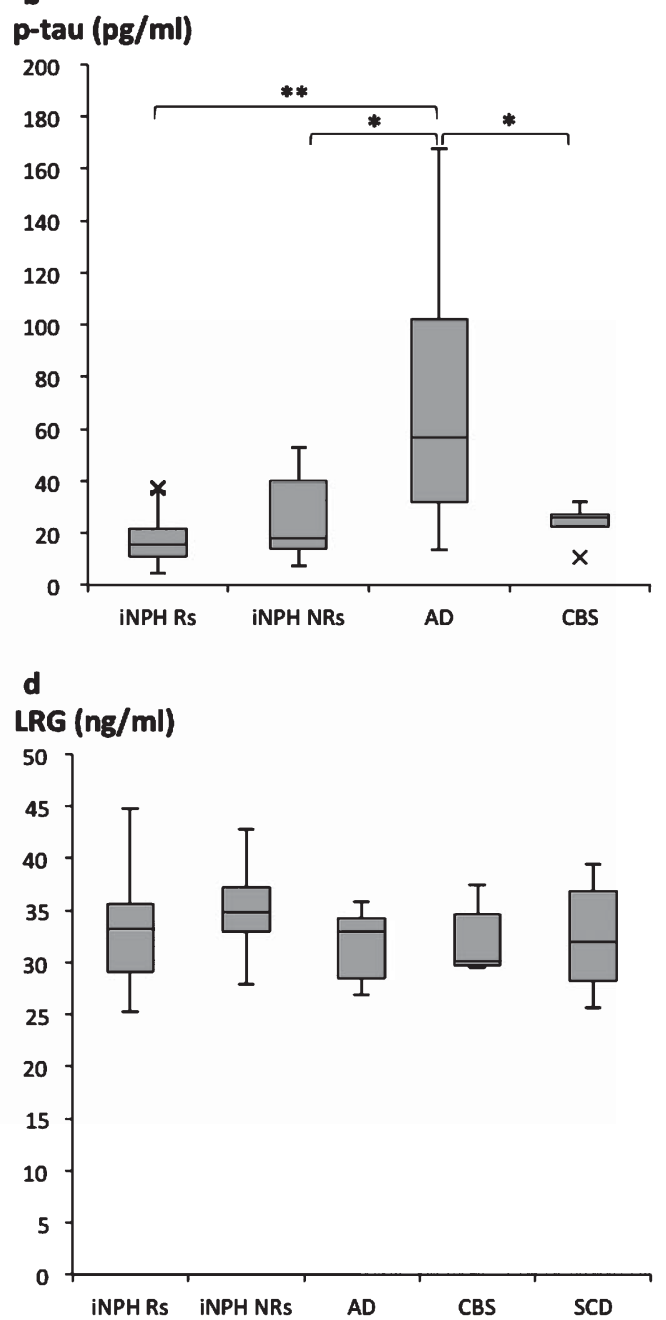

Fig. 1. Box plots of each CSF biomarker in the studied group; t-tau (a), p-tau (b), A $\beta_{42 / 40}$ ratio (c), and LRG (d). Horizontal lines within boxes show median values, boxes show upper and lower interquartile (IQ) ranges, whiskers indicate the 1.5 times IQ, a cross (x) in the figure indicate outlier values (1.5 times IQ). ${ }^{*} p<0.05,{ }^{* *} p<0.001$. 


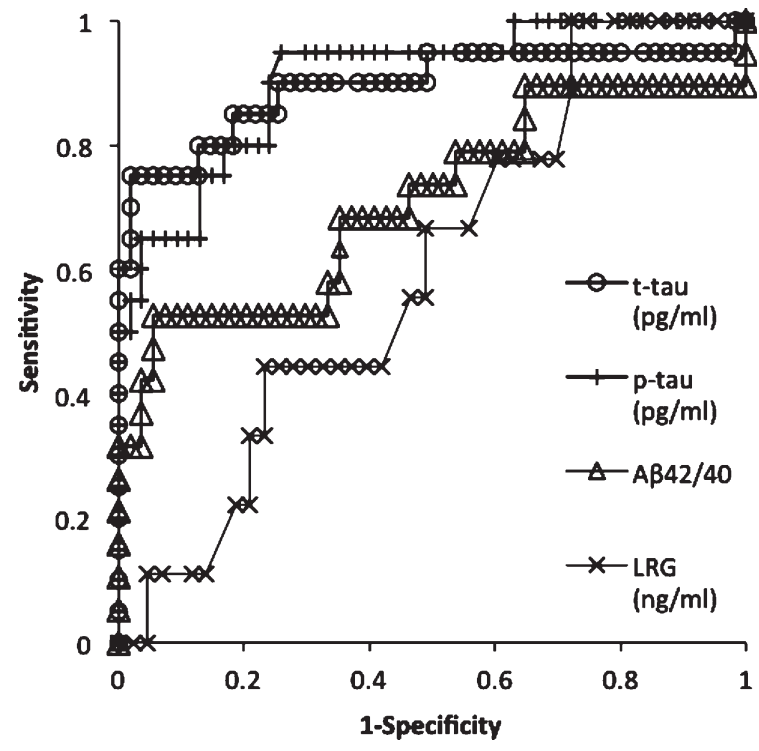

Fig. 2. ROC curve analysis for each CSF biomarker for differentiation of iNPH from AD.

Table 2

Comparison of CSF biomarkers between patients with iNPH and AD: ROC analysis

\begin{tabular}{lcccc}
\hline & t-tau & $\mathrm{p}$-tau & $\mathrm{A} \beta_{42 / 40}$ & $\mathrm{LRG}$ \\
\hline AUC & 0.90 & 0.91 & 0.71 & 0.60 \\
Cutoff value & $766 \mathrm{pg} / \mathrm{ml}$ & $24.4 \mathrm{pg} / \mathrm{ml}$ & 0.038 & $35.8 \mathrm{ng} / \mathrm{ml}$ \\
Sensitivity & $75 \%$ & $95 \%$ & $53 \%$ & $100 \%$ \\
Specificity & $98 \%$ & $74 \%$ & $94 \%$ & $28 \%$ \\
\hline
\end{tabular}

iNPH, idiopathic normal pressure hydrocephalus; AD, Alzheimer's disease; t-tau, total tau; $p$-tau, phosphorylated tau; $A \beta$, amyloid$\beta$; LRG, leucine-rich $\alpha$-2-glycoprotein; ROC, receiver operating characteristic analysis; AUC, area under the curve.

subgroups compared to iNPH Rs $(\mathrm{R}=0.54)$ subgroup (Fig. 3a).

The $\mathrm{A} \beta_{42 / 40}$ ratio was higher in iNPH patients than in AD patients $(p<0.01)$. Using ANCOVA with diagnostic group of iNPH and AD as a factor, and MMSE scores as a covariate, the diagnostic group did not show impact on the levels of $\mathrm{A} \beta_{42 / 40}$ ratio ( $p=0.08$ ) (Fig. 1c, Table 1). MMSE scores of AD patients were correlated with the levels of $A \beta_{42}(R=0.44)$ and inversely correlated to the levels of $t$-tau $(\mathrm{R}=0.37)$, whereas the iNPH group showed less clear correlations between MMSE scores and the levels of $A \beta_{42}(R=0.28)$. In iNPH group, MMSE scores and the levels of $t$-tau were not inversely correlated as in $\mathrm{AD}(\mathrm{R}=0.17)$ (Fig. 4a, b). The levels of $A \beta_{40}$ and $A \beta_{42}$ showed strong correlations in the iNPH $(\mathrm{R}=0.73)$ and $\mathrm{CBS}(\mathrm{R}=0.94)$ groups, while they showed less clear correlation in

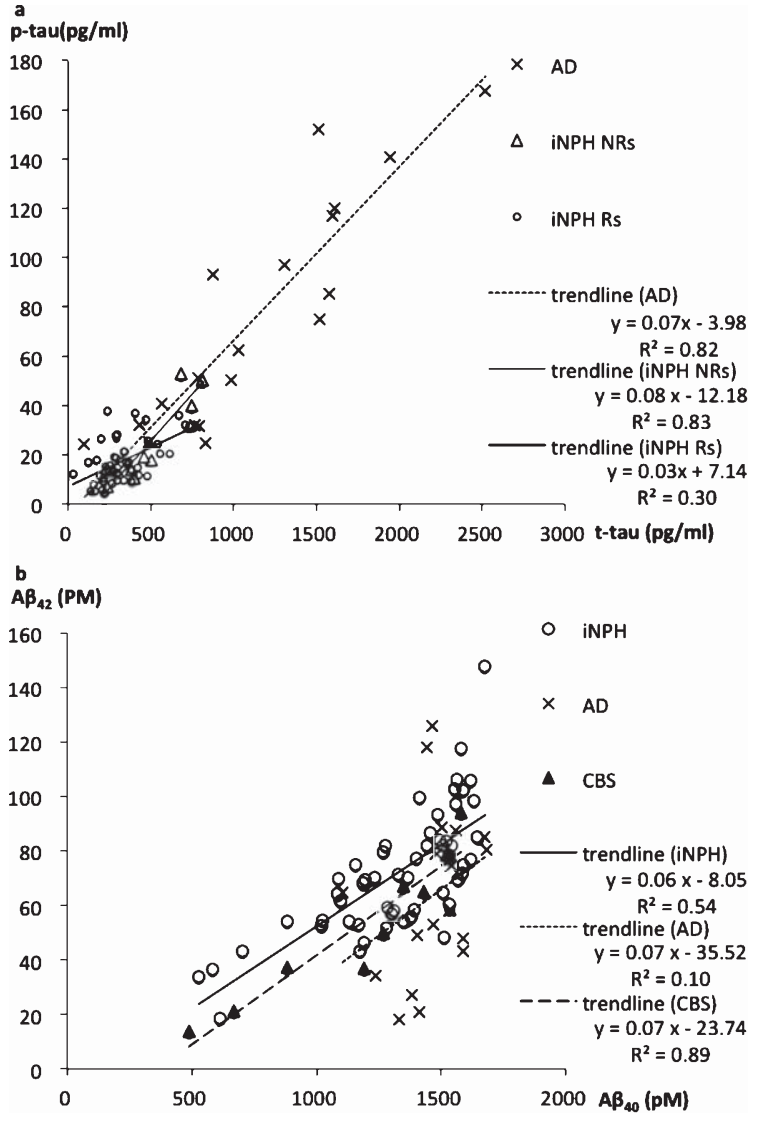

Fig. 3. Correlation between t-tau and p-tau in iNPH Rs, NRs, and $\mathrm{AD}$ (a). Correlation between $\mathrm{A} \beta_{40}$ and $A \beta_{42}$ in iNPH, $A D$, and $C B S$ (b).

the AD group ( $\mathrm{R}=0.26)$ (Fig. 3b). LRG levels did not differ among iNPH, AD, CBS, and SCD (Fig. 1d).

\section{DISCUSSION}

In this study, t-tau and p-tau levels were highly decreased in iNPH patients than in AD patients. Levels of t-tau and p-tau showed a strong correlation each other in AD, while they did not in iNPH Rs subgroup. Given that these two pathological conditions are distinguishable by differential biomarker profiles, iNPH appears to have a unique tau-negative pathology that differs from AD. In iNPH, an excessive volume of CSF, mainly accumulating in the cerebral ventricles and subarachnoid space of cortical sulci, might dilute the concentration of tau in CSF, which might account for the lower tau values. In iNPH Rs, t-tau levels were lower than those in NRs. Therefore, iNPH patients who have lower t-tau concentration in CSF might be the better candidates for surgical treatment. 


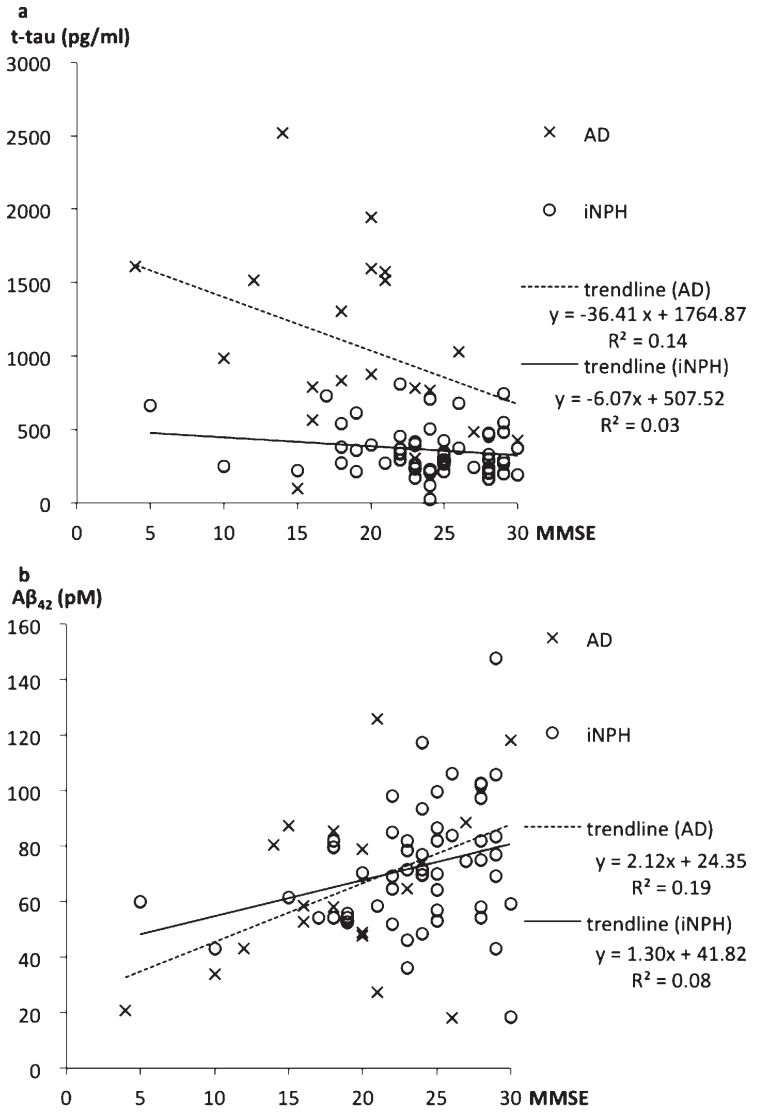

Fig. 4. Correlations between the MMSE score and t-tau (a) and $A \beta_{42}$ (b) in iNPH and AD.

The iNPH patients with higher t-tau and p-tau levels might have stronger overlaps of tau pathology such as AD. Another possibility is that the iNPH itself has progressed to an irreversible stage due to a longer clinical course, leading to severe white matter degeneration and neurodegeneration with higher average $\mathrm{t}$-tau levels in CSF. Thus, iNPH patients with higher CSF t-tau values might have a progressive clinical course even after shunt surgery.

CSF $A \beta_{42 / 40}$ ratio in iNPH patients seemed to be significantly higher than in AD patients. We found a correlation between MMSE scores of iNPH patients and the levels of $A \beta_{42}$, which might suggest that the cognitive function of iNPH patients is affected by the preclinical stages of AD [14]. As there was a nonnegligible MMSE score difference between the iNPH and AD groups $(p=0.001)$, we used ANCOVA with MMSE scores as a covariate for the evaluation of CSF $A \beta_{42 / 40}$ ratio between the two groups, resulting that the CSF $A \beta_{42 / 40}$ ratio does not statistically differ between iNPH and AD patients.
Interestingly, the profile in the levels of $A \beta_{40}$ and $A \beta_{42}$ in the $A D$ group was different from those in the iNPH and CBS groups. In the groups of iNPH and CBS, the levels of $A \beta_{40}$ and $A \beta_{42}$ showed positive correlation; individuals with high $A \beta_{40}$ value in the CSF showed high $A \beta_{42}$ value as well. However, this correlation was not so clear in $\mathrm{AD}$ group. This might be explained by the preference for $A \beta_{42}$ deposition in the cerebral cortex of AD brain. The profile of $A \beta_{40}$ and $\mathrm{A} \beta_{42}$ in iNPH group was similar to that in CBS group. This suggests that the amyloid pathology of iNPH as a group is discrete from that of $\mathrm{AD}$ as a group. The pathophysiology of iNPH does not seem to favor $A \beta_{42}$ deposition like AD.

The levels of LRG in CSF are reported to be increased in iNPH patients [11]. However, LRG levels were not different between iNPH and AD patients in this study. We also evaluated LRG levels between Rs and NRs iNPH patients, but could not find any difference. Since LRG is reported to be a potential biomarker for the diagnosis of iNPH, larger size of studies are required to evaluate the previously reported results.

The goal of our study is to search for useful CSF biomarkers to differentiate iNPH from AD. However, the comparison of CSF biomarker between iNPH and age-matched healthy control subjects was more ideal. Unfortunately, because the subjects were elderly patients, access to CSF samples from pure normal agematched control subjects were limited in this study. The groups compared as control consisted of CBS and SCD. We chose to compare CBS patients because their levels of CSF tau are reported to not be increased [15], and they are not specific for amyloid pathology. Therefore, we assume that the quality of the compared groups including CBS were suitable for investigation of the biomarkers described above.

As iNPH NRs had higher CSF t-tau compared to Rs, and the cognitive function of iNPH patients was mildly associated with the level of CSF $A \beta_{42}$, it is true that some individuals in iNPH overlap with other neurodegenerative diseases, especially AD. The degree of overlapping pathology, which is clinically ineludible but difficult to be estimated, might be reflected by CSF biomarkers to a certain extent. The overlaps in CSF biomarker profile between these distinct pathological conditions may suggest that the pathophysiology of each iNPH individual is sometimes mixed and heterogeneous.

However, in conclusion, our results show that t-tau and p-tau were equally and highly useful CSF biomarkers for differentiating iNPH from AD, even though they might not specifically reflect the iNPH 
pathophysiology. In addition, as in the two groups, the difference of $A \beta$ profile shows discrete pathologies. The above biomarkers reveal that iNPH has a different CSF biomarker profile from AD. These CSF biomarkers could improve the diagnostic accuracy of iNPH, especially iNPH Rs with less tau pathology. They could also influence selection of the treatment course and could predict the prognosis of patients. The quest for more specific CSF biomarkers for iNPH is ongoing, and further studies should be conducted with in a larger population of patients.

\section{ACKNOWLEDGMENTS}

This work was supported in part by grants-in-aid from the Ministry of Education, Culture, Sports, Science and Technology of Japan, and the SRF (S.S.) and by an A-STEP grant from the Japan Science and Technology Agency (A.K.).

Authors' disclosures available online (http://j-alz. com/manuscript-disclosures/14-2622).

\section{REFERENCES}

[1] Adams RD, Fisher CM, Hakim S, Ojemann RG, Sweet WH (1965) Symptomatic occult hydrocephalus with "normal" cerebrospinal-fluid pressure. A treatable syndrome. $N$ Engl J Med 273, 117-126.

[2] Ray B, Reyes PF, Lahiri DK (2011) Biochemical studies in Normal pressure hydrocephalus (NPH) patients: Change in CSF levels of amyloid precursor protein (APP), amyloid-beta (Abeta) peptide and phospho-tau. J Psychiatr Res 45, 539547.

[3] Cabral D, Beach TG, Vedders L, Sue LI, Jacobson S, Myers K, Sabbagh MN (2011) Frequency of Alzheimer's disease pathology at autopsy in patients with clinical normal pressure hydrocephalus. Alzheimers Dement 7, 509-513.

[4] Jack CR Jr, Vemuri P, Wiste HJ, Weigand SD, Lesnick TG, Lowe V, Kantarci K, Bernstein MA, Senjem ML, Gunter JL, Boeve BF, Trojanowski JQ, Shaw LM, Aisen PS, Weiner MW, Petersen RC, Knopman DS, Alzheimer's Disease Neuroimaging, Initiative (2012) Shapes of the trajectories of 5 major biomarkers of Alzheimer disease. Arch Neurol 69, 856-867.

[5] Seppala TT, Koivisto AM, Hartikainen P, Helisalmi S, Soininen H, Herukka SK (2011) Longitudinal changes of CSF biomarkers in Alzheimer's disease. J Alzheimers Dis 25, 583594.
[6] Hu WT, Chen-Plotkin A, Arnold SE, Grossman M, Clark CM, Shaw LM, McCluskey L, Elman L, Karlawish J, Hurtig HI, Siderowf A, Lee VM, Soares H, Trojanowski JQ (2010) Biomarker discovery for Alzheimer's disease, frontotemporal lobar degeneration, and Parkinson's disease. Acta Neuropathol 120, 385-399.

[7] Bech-Azeddine R, Hogh P, Juhler M, Gjerris F, Waldemar G (2007) Idiopathic normal-pressure hydrocephalus: Clinical comorbidity correlated with cerebral biopsy findings and outcome of cerebrospinal fluid shunting. J Neurol Neurosurg Psychiatry 78, 157-161.

[8] Kapaki EN, Paraskevas GP, Tzerakis NG, Sfagos C, Seretis A, Kararizou E, Vassilopoulos D (2007) Cerebrospinal fluid tau, phospho-tau181 and beta-amyloid1-42 in idiopathic normal pressure hydrocephalus: A discrimination from Alzheimer's disease. Eur J Neurol 14, 168-173.

[9] Agren-Wilsson A, Lekman A, Sjoberg W, Rosengren L, Blennow K, Bergenheim AT, Malm J (2007) CSF biomarkers in the evaluation of idiopathic normal pressure hydrocephalus. Acta Neurol Scand 116, 333-339.

[10] Nakajima M, Miyajima M, Ogino I, Watanabe M, Miyata H, Karagiozov KL, Arai H, Hagiwara Y, Segawa T, Kobayashi $\mathrm{K}$, Hashimoto Y (2011) Leucine-rich alpha-2-glycoprotein is a marker for idiopathic normal pressure hydrocephalus. Acta Neurochir (Wien) 153, 1339-1346; discussion 1346.

[11] Li X, Miyajima M, Mineki R, Taka H, Murayama K, Arai H (2006) Analysis of potential diagnostic biomarkers in cerebrospinal fluid of idiopathic normal pressure hydrocephalus by proteomics. Acta Neurochir (Wien) 148, 859-864; discussion 864.

[12] Relkin N, Marmarou A, Klinge P, Bergsneider M, Black PM (2005) Diagnosing idiopathic normal-pressure hydrocephalus. Neurosurgery 57, S4-16; discussion ii-v.

[13] McKhann G, Drachman D, Folstein M, Katzman R, Price D, Stadlan EM (1984) Clinical diagnosis of Alzheimer's disease: Report of the NINCDS-ADRDA Work Group under the auspices of Department of Health and Human Services Task Force on Alzheimer's Disease. Neurology 34, 939-944.

[14] Sperling RA, Aisen PS, Beckett LA, Bennett DA, Craft S, Fagan AM, Iwatsubo T, Jack CR Jr, Kaye J, Montine TJ, Park DC, Reiman EM, Rowe CC, Siemers E, Stern Y, Yaffe K, Carrillo MC, Thies B, Morrison-Bogorad M, Wagster MV, Phelps CH (2011) Toward defining the preclinical stages of Alzheimer's disease: Recommendations from the National Institute on Aging-Alzheimer's Association workgroups on diagnostic guidelines for Alzheimer's disease. Alzheimers Dement 7, 280-292.

[15] Arai H, Morikawa Y, Higuchi M, Matsui T, Clark CM, Miura M, Machida N, Lee VM, Trojanowski JQ, Sasaki H (1997) Cerebrospinal fluid tau levels in neurodegenerative diseases with distinct tau-related pathology. Biochem Biophys Res Commun 236, 262-264. 